\title{
Genetic structure and haplotype diversity of Tachypleus gigas population along the west coast of peninsular Malaysia-inferred through mtDNA AT rich region sequence analysis.
}

\begin{abstract}
A detailed investigation was carried out to determine the genetic structure and haplotype diversity of Malaysian horseshoe crab (Tachypleus gigas [Muller, 1785]) distributed along the west coast of Peninsular Malaysia. Mitochondrial DNA (AT rich region $=369 \mathrm{bp}$ ) analysis showed that $\mathrm{T}$. gigas had 13 haplotypes along the Malaysian west coast of which 4 were unique to Selangor samples while 3 were unique to Johor sample and 1 each were unique to other two stations respectively. Highest haplotype diversity (h) was observed among the Selangor samples $(0.873 \pm 0.071)$ followed by Langkawi, Johor and Kedah samples with $0.833 \pm 0.222,0.752 \pm 0.066$ and $0.733 \pm 0.155$ values, respectively. Over all haplotype diversity of T. gigas in west coast of Malaysia was observed to be $0.797 \pm 0.129$. Pair wise haplotype frequency $(\mathrm{FST})$ value were statistically significant $(\mathrm{p}<0.05)$ for all the groups except for Langkawi/Kedah samples indicating higher gene flow (Lower haplotype diversity) among these two populations. Average nucleotide diversity $(\pi)$ was higher in Selangor samples $(0.0083 \pm 0.001)$ followed by Johor $(0.0063 \pm 0.0011)$ and it was almost similar in Langkawi $(0.0045 \pm 0.0012)$ and Kedah $(0.0040 \pm 0.0008)$ samples which indicated higher polymorphic sites in Selangor and Johor samples while it was lower in Langkawi and Kedah samples. In addition phylogenetic analysis clearly clustered T. gigas samples from $\mathrm{T}$. tridentatus samples indicating good phylogenetic signals in mtDNA AT rich region. Overall, findings from this study have important implications for proper management and conservation of this living fossil along the west coast of Peninsular Malaysia.
\end{abstract}

Keyword: Haplotype diversity; Nucleotide diversity; mtDNA AT rich region; Tachypleus gigas; Genetic conservation. 